\title{
Desmatamento na Amazônia Brasileira: Com que intensidade vem ocorrendo? (*)
}

\author{
Philip M. Fearnside $(* *)$
}

\begin{abstract}
Resumo
O desmatamento na Amazônia brasileira está ocorrendo a uma taxa inédita. Uma série de imagens de satélite LANDSAT de áreas de colonização em Rondônia, de 1973 até 1978 , indicam que as tendências de desmatamento podem ser exponenciais, porém dados são escassos demais para conclusões firmes. Informações disponíveis de imagens LANDSAT de 1975 e 1978. com cobertura de todos os $5 \times 10^{6} \mathrm{~km}^{2}$ da Amazônia Legal brasileira, possibilitam o cálculo do que aconteceria se o desmatamento ocorresse exponencialmerto nas taxas indicadas pelas imagens destas duas detas. A não homogeneidade das taxas de desmatamento resultaria em que algumas áreas seriam desmatadas mais cedo e outras, mais tarde; se as taxas de desma tamento permanecessem constantes, dentro de cada Estado ou Território, estes seriam desmatados em datas que variam desde 1988 para Rondônia até 2159 para Amapá. Esclareça-se que nenhum destes cálculos representa uma previsão do que está esperado a acontecer, mas apenas cenários hipotéticos do que aconteceria, caso estas taxas fossem sustentadas ao longo do tempo. O desmatamento é um processo complexo demais para ser adequadamente representado por equa. ções simples.
\end{abstract}

\section{INTRODUÇÃO: ESTIMATIVAS CONFLITANTES}

Variam grandemente as estimativas do quanto da Floresta Tropical Úmida da Amazônia Brasileira tem sido desmatado e quão rápido é este processo. Ainda mais impressionantes são as diferenças de interpretação de quão rápido será o desmatamento da floresta no futuro e se as tendências atuais são causa de preocupação. É importante examinar, nas taxas atuais, a informação a que temos acesso e as tendências prováveis para a região come um todo, e examinar mais detalhadamente o processo de desmatamento num dos correntes focos da atividade de derrubada: o Estado de Rondônia.
Oficiais do Instituto Brasileiro de Desenvolvimento Florestal (IBDF), da Superintendência do Desenvolvimento da Amazônia (SUDAM) e de outrcs órgãos ofereceram estimativas da derrubada da Amazônia, resumidas na Tabela 1. Em janeiro de 1980 o Instituto Nacional de Pesquisas Espaciais (INPE) liberou as primeiras estimativas para a região como um todo, baseado na interpretaçäo das imagens do satélite LANDSAT (Tabela 1). As descobertas do INPE que apenas $1,55 \%$ da Amazônia Legal Brasileira tinha sido desmatada, no tempo das imagens de 1978, levou alguns, a uma certa complacência com respeito a desmatamento na regiäo. Um exame mais aproximado das tendências indicados nos dados do INPE, ao nível de região, assim como meu próprio trabalho com as informações do LANDSAT sobre Rondônia, não deixam espaço para tal complacência.

Alguns probiemas com imagens LANDSAT conduzem a subestimativa de desmatamento. Estes problemas incluem a exclusão de desma. tamentos "muito pequenos" (Tardim et al., 1980). E o que é mais importante, é difícil distinguir a floresta primária de uma capoeira com mais de que uns poucos anos de vida. Desenhos a partir de imagens LANDSAT sobre Rondônia, que serão discutidas mais adiante neste artigo, indıcam que vários desmatamentos em 1976 voltaram a ser classificados como floresta virgem em 1978. No caso do estudo do INPE (Tardin et al., 1980) a diferença fica mais clara no que se refere ao desmatamento conhecido na Zona Bragantina. Esta área do Pará, de aproximadamente $30.000 \mathrm{~km}^{2}$ próxima à cidade de Bragança, tem sido ocupada desde o fim do séc. XIX, e encontra-se virtual e completamente desmatada há muitos anos (Penteados 1967; Sioli, 1973: 327). Minhas próprias

(*) - Versão original inglesa publicada em Interciencia, 7 (2): 82-88, 1982.

(*) - Instituto Nacional de Pesquisas da Amazônia. Manaus. 
TABELA 1 - Estimativas do desmatamento da floresta na Amazônia Brasileira

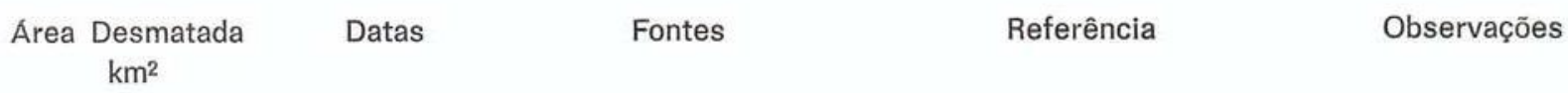

Dados para a Amazônia Legal $\left(4.975 .527 \mathrm{~km}^{2}\right)$

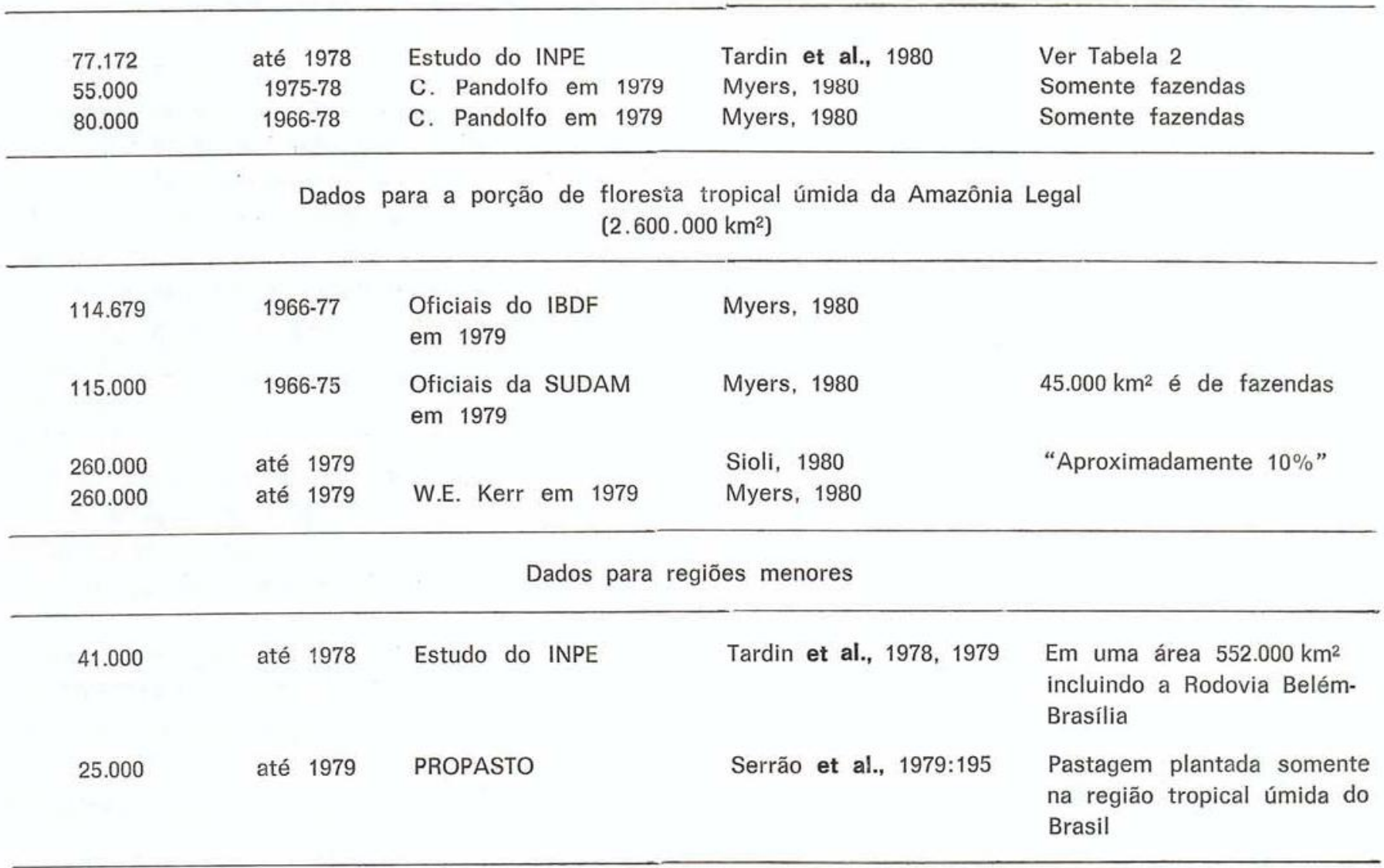

Taxas de desmatamento

\begin{tabular}{|c|c|c|c|c|}
\hline $100.000 \mathrm{~km}^{2} / \mathrm{ano}$ & 1978 & $\begin{array}{l}\text { O. Bittencourt, } \\
\text { RADAMBRASIL em } \\
1978\end{array}$ & Sioli, 1980:261 & Amazônia Legal \\
\hline $100.000 \mathrm{~km}^{2} / \mathrm{ano}$ & 1975 & "Fotos de satélite" & $\begin{array}{l}\text { Jornal do Brasil (Rio de } \\
\text { Janeiro), } 26 \text { de julho de } \\
1977 \text { citado por Mahar, } \\
\text { 1979:137 }\end{array}$ & Amazônia Legal \\
\hline $10.000 \mathrm{~km}^{2} /$ ano & $\begin{array}{l}1960-75 \\
\text { média }\end{array}$ & $\begin{array}{l}\text { Estudo do FAO/IBDF } \\
1975\end{array}$ & $\begin{array}{l}\text { Muthoo, 1977, citado } \\
\text { por Reis, 1978:8 }\end{array}$ & $\begin{array}{l}\text { Floresta Amazônica } \\
\text { brasileira }\end{array}$ \\
\hline $10.427 \mathrm{~km}^{2} /$ ano & $\begin{array}{l}\text { 1966-77 } \\
\text { média }\end{array}$ & $\begin{array}{l}\text { Oficiais do IBDF } \\
\text { em } 1979\end{array}$ & Myers, $1980: 128$ & $\begin{array}{l}\text { Área de floresta tropical } \\
\text { úmida }\end{array}$ \\
\hline $12.777 \mathrm{~km}^{2} /$ ano & $\begin{array}{l}\text { 1966-75 } \\
\text { média }\end{array}$ & $\begin{array}{l}\text { Oficiais da SUDAM } \\
\text { em } 1979\end{array}$ & Myers, 1980:128 & $\begin{array}{l}\text { Area de floresta tropical } \\
\text { úmida }\end{array}$ \\
\hline
\end{tabular}

\footnotetext{
Abreviaturas: INPE - Insttiuto Nacional de Pesquisas Espaciais

IBDF - Instituto Nacional de Desenvolvimento Florestal

SUDAM - Superintendência do Desenvolvimento da Amazônia

RADAMBRASIL - Projeto Radar da Amazônia e o Brasil

PROPASTO - Programa de Pastagens

FAO - Organização das Nações Unidas para Alimentação e Agricultura
} 
visitas a esta área de 1975 em diante confir. mam a impressão de que não permaneceu nada da fioresta original. Somiente a área da Zona Bragantina é maior do que os $28.595 \mathrm{~km}^{2}$ indicados pelo estudo do INFE (Tardin et ai.. 1980) como sendo desmatada até 1975 em toda a Amazônia Legal, e é quase quatro vezes os $8.654 \mathrm{~km}^{2}$ de área indicada como desmatada até 1975 no Estado do Pará.

Mais importante do que subestimar as áreas desniatadas dadas as limitaçöes técnicas de interpretação do LANDSAT é a taxə em que este desmatamento vem crescendo, e as forças em movimento levando a um provável maior crescimento futuro. A constatação básica do INPE, ou seja que a área desmatada em 1978 era pequena em relação à área da Amazônia Lega!, é bastante correta, mesmo considerando-se alguma subestimativa nas medidas de área. Várias indicações sugerem que as tendências recentes de desmatamento podem ser exponenciais, ainda que as informações a que se tem acesso sejam insuficientes para tirarem-se conclusões definitivas. Estas tendências colidem com uma das maiores ilusões da Amazônia: a ilusão de seu tamanho infinito

A NATTUREZA DO CRESCIMENTÓ EXPONENCIAL: ILUSÃO DE TAMANHO

A taxa pela qual uma pequena quantidade pode tornar-se maior, quando crescendo exponencialmente, sempre foi uma fonte de sur. presas para as pessoas, desde os consumidores de supermercado até os planejadores do governo. O caso da inflação é o melhor exemplo. No Brasil, a inflação tem estado na categoria de 2 dígitos durante quase todo o tempo recordável pela população $d \in$ hoje, e, mais recentemente, na categoria de 3 dígitos. Ainda assim os consumidores são continuamente surpreendidos pela magnitude dos aumentos quando fazem compras semanais. A idéia de que a pessoa mais velha tenha comprado uma casa por menos do que o preço atual de uma garrafa de Coca-Coia ainda causa espanto, mesmo depois de uma vida exposta à tendência exponencial. A mesma dificuldade atrapa-
Iha a visualização do crescimento de uma área desmatada relativamente pequena até ocupar a vasta extensäo da Amazônia. Uma tendência exponencial pode levar justamente a um tal crescimento em muito poucos anos. Vale a pena observar mais de perto algumas das propriedades de padrões exponericiais em geral, e como estes săo ligados ao desmatamento na Amazônia .

Quantidades que crescem por quantias que dependem da quantia já presente, aumentam. exponencialmente. Tal padrão aplica-se a uma variedade diversa de fenômenos, desde dinheiro em contas bancárias a populações irrestritas de organismos, e pode enquadrar-se também à tendência corrente do desmatamento da Amazônia. Se uma quantidade cresce por uma fração constante durante cada intervalo de tempo, seu tamanho a qualquer instante pode ser descrito por:

$$
\mathrm{N}_{\mathrm{t}}=\mathrm{N}_{\mathrm{o}}(1+\mathrm{i})^{\mathrm{t}} \text { Equação } 1 .
$$

onde: $\quad \mathrm{N}_{\mathrm{t}}=\mathrm{o}$ número no tempo " $\mathrm{t}$ "

$\mathrm{No}_{\mathrm{o}}=\mathrm{o}$ número inicial (número no tempo " 0 ")

$\mathrm{i}=$ a proporção pela qual o número cresce em cada unidade de tempo $(1+i=a$ taxa finita de crescimento)

$\mathrm{t}=\mathrm{o}$ número de unidades de tempo decorridas.

A Equação 1 descreveria, por exemplo. o crescimento do dinheiro numa conta bancária onde os juros são creditados somente uma vez por ano, se $\mathrm{t}=1$ ano $\mathrm{e} \mathrm{i}=\mathrm{a}$ taxa de juros expressa como uma proporção. O dinheiro numa conta bancária cresce mais rapidamente quando aumenta o número de vezes no ano em que os juros são creditados. Incluindo o número de vezes em que os juros são creditado (q vezes / unidade de tempo), teremos a forma de equação familiar aos banqueiros :

$$
\mathrm{N}_{\mathrm{t}}=\mathrm{N}_{\mathrm{o}}\left(1+\frac{\mathrm{i}}{\mathrm{q}}\right)^{\mathrm{t} \mathrm{q}} \quad \text { Equação } 2 .
$$

$\mathrm{Na}$ nsedida em que o número de vezes os juros são creditados, aproxima-se do infinito, a 
Equação 2 aproxima a equação exponencial na forma familiar aos biólogos (ver Pielou, 1974: 360 para uma derivação):

$$
\mathrm{N}_{\dot{\mathrm{x}}}=\mathrm{N}_{0}^{\mathrm{rt}} \text { Equação } 3 .
$$

onde:

$$
\begin{aligned}
& r=a \text { taxa de crescimento inata (rela- } \\
& \text { In } \left.(1+i) \text { ou } \mathbf{i}=e^{r}-1\right) \\
& a=a \text { base dos logaritmos naturais (a } \\
& \text { constante } 2,71828 . . \text { ). }
\end{aligned}
$$

A Equação 3 é a melhor representante de fenômenos tais como crescimento populacional e desmatamento, dado a representação do crescimento exponencial como um processo contínuo ao em vez de uma série de eventos não contínuos como o juro creditado no final de cada intervalo de tempo (como nas Equações 1 e 2). A diferença nos resultados obtidos usando uma representação não contínua (Equação 1 e 2) ao em vez de uma contírua (Equação 3) pode ser relativamente pequeno para valores pequenos de " $\mathrm{i}$ " ou " $r$ ", mas aumentam na medida en: que as taxas aumentam. A escolha apropriada da equação depende da natureza do fenômeno e a maneira para cierivar o valor da taxa empregada no cálculo.

A taxa de crescimento exponencial ( $r$ ) pode ser obtido refazendo a Equação 3:

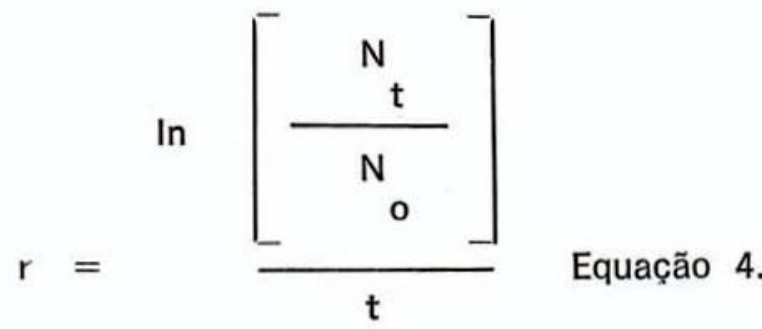

Para calcular a taxa a partir de informação referente a um dado intervalo, os valores de " $\mathrm{N}_{\mathrm{o}}$ " e $\mathrm{N}$ " usados representariam o total de uma quantidade no começo e fim do intervalo, e " $t$ " o tempo decorrido.

C tempo necessário para destruir um recurso, por exemplo, desmatar uma área dada comc a Amazônia Legal Brasileira, poderá ser obtido refazendo a Equação 4 :

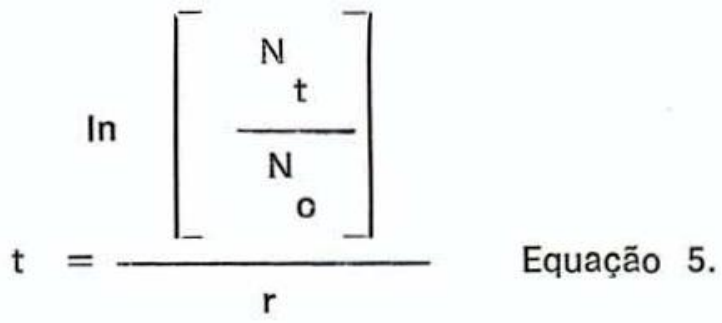

O tempo necessário, a partir do presente para desmatar uma região, por exemplo, será dado por " $\mathrm{t}$ " na Equação 5 se " $\mathrm{N}_{\mathrm{t}}$ " for a área tota! da região e " $\mathrm{N}_{\mathrm{o}}$ " a quantidade desmatada até o presente.

A dificuldacie em visualizar grandes resultados de mudanças exponenciais de valores, aparentemente pequenos, da taxa exponencial de crescimento ( $r$ ), faz com que seja conveniente converter estas taxas em tempo de duplicação. O tempo necessário para duplicar um resultado pode ser obtido da Equação 5 substituindo o valor 2 pela razão $N_{i} / N_{0}$. O tempc de duplicação será, então, igual a In $2 / r$, ou $0,6931 / r$. Para realizar cálculos mentais mais rápidos é mais fácil estimar o tempo de duplicação em anos dividindo o valor 70 (uma aproximação de 69,31) pela porcentagem de crescimento anual.

DESMATAMENTO DA FLORESTA TROPICAL ÚMIDA: IMPLICAÇÕES DA TENDÊNCIA EXPONENCIAL

A tendência do desmatamento recente parece ser exponencial, ainda que as informações disponíveis não possam ser consideradas conclusivas. Admitindo-se que tal tendência continua no futuro numa taxa constante ( $r$ " da Equação 3), podemos observar as conseqüências da tendência. A solidez $\epsilon$ a fraqueza desta suposição quanto a uma taxa de crescimento consiante será discutida mais adiante.

C estudo do INPE (Tardin et al., 1980) indica que $28.595,25 \mathrm{~km}^{2}$ da Amazônia Legal foi desmatada até 1975 , aumentando este desmatamento para $77.171,75 \mathrm{~km}^{2}$ até 1978 . Usando estes valores para " $\mathrm{N}_{\mathrm{o}}$ " e " $\mathrm{N}_{\mathrm{t}}$ " respectivamente na Equação 4, e os três anos de interva- 
lo de observação para " $t$ ", podemos calcular um valor para a taxa anual de crescimento " $r$ " de 0,33093 . Observe que isto corresponde a um tempo de duplicação de somente 2,09 anos. O tempc que se necessita para desmatar toda a Amazônia Legal a esta taxa pode ser obtido da Equação 5, substituindo o valor acima por "r" o 4.975.527 km² de área da Amazônia Legal por " $\mathrm{N}_{\mathrm{t}}$ " e a estimativa de desmatamento até 1978 de $77.171,75 \mathrm{~km}^{2}$ por " $\mathrm{N}_{\mathrm{o}}$ ". O tempo resultante para desmatar a área é de 12.6 anos, o que quer dizer que o tempo pode vir a ser completado até o ano 1991.

A concentração da atividade de derrubada em certas regiões tem sido im importante aspecto do padrão de desmatamento. A derrubada mais intensa tem-se dado em Rondônia, Acre c nas áreas das fazendas de gado do sul do Pará, oeste de Maranhão, norte de Goiás e Mato Grosso. A Tabela 2 mostra as implicações de um padrão de crescimento exponencial por Estado ou Território, calculado, a partir dos dados do INPE (Tardin et al., 1980: 12).
Vale a pena repetir que os tempos para o desmatamento completo mostrados na Tabela 2 assumem uma " $r$ " constante na equação exponencial. O valor de " $r$ ", sem dúvida, não é constante: pode mudar positiva ou negativamente. A tabela, portanto, não representa uma previsão do que irá ocorrer na realidade. mas mostra as conseqüências do prolongamento da tendência exponencial.

\section{RONDÔNIA: UM LABORATÓRIO PARA O ESTUDO Do DESMatamento}

Rondônia, como pode ser visto na Tabela 2, é o Estado com a mais rápida taxa de desma. tamento na Amazônia Legal, com uma tendência exponencial na taxa atual levando no desmatamento completo até 1988 ('). Dentro de Roncionia, certas áreas mostraram desmatamento verdadeiramente explosivo. O Município de Cacoal, por exemplo, teve uma área desmatada de 2.150 ha em 1975, aumentando para 66.950 ha em 1978 (Brasil, CNPq-INPE,

\section{TABELA 2 - Desmatamento na Amazônia Legal assumindo a tendência exponencial}

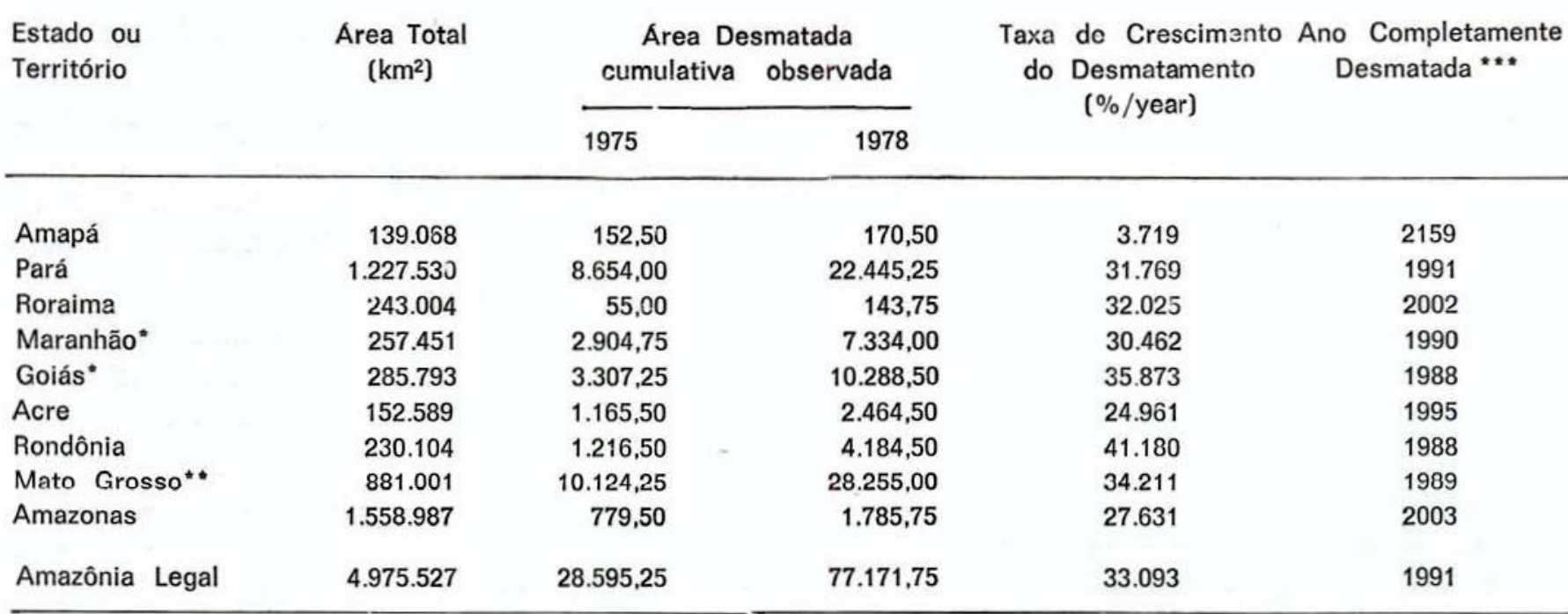

(*) - Estodos incluidos não totalmente dentro đạ Amazônia Legal: Goiás ao norte de 130 de latitude S. e Maranhăo ao oeste $44^{\circ}$ longitude 0 .

(*) - Mato Grosso é o novo Estado criado em 1ọ de Janeiro de 1979 da parte norte do antigo Estado de Mato Grosso.

$(* \star *)$ - Éstes valores não são prediçōes de eventos futuros. Veja texto para explicaçōes.

(1) - Valores para o desmatamento e a área total de Rondônia que diferem ligeiramente dos de Tardin et al. (1980) apresentados na Tabela 2 foram proporcionados pelo Brasil, CNPq-INPE, Departamento de Sensoriamento Remoto, Divisão de Aquisição de Dados, Seção de Aeronave e Apoio Cartográfico (1980, citado por Calvente, 1980:27): área total $243.004 \mathrm{~km}^{2}$, desmatada a:é $19751.216,50 \mathrm{~km}^{2} \mathrm{e}$ até $19784.162,00 \mathrm{~km}^{2}$. Ambos os conjuntos de dados leva ao desmatamento até 1988, assumindo uma tendência exponencial. 
Departamento de Sensoriamento Remoto, Divisão de Aquisição de Dados, Seção de Aeronave e Apoio Cartográfico, 1980 citado por Calvente, 1980:27). Um crescirnento exponencial a esta taxa teria um valor " $r$ " de 1,14616 ou um tempo de duplicação de 0,6 anos! A área total de 80.000 ha do Município (Brasil, Território Federal de Rondônia, Secretaria de Agricultura, 1980: Vol. 2 p. 37) teria sido comple. tamente desmatada até o final de 1981 se esta taxa tivesse permanecido inalterada. Dentro do Município, a área desmatada do sudoeste da rodovia BR-364 cresceu ainda mais rapidamente, dos 550 ha em 1975 para os 24.875 ha em 1978 (Brasil, CNPq-INPE, Departamento de Sensoriamento Remoto, Divisão de Aquisi. ção de Dados, Seção de Aeronave e Apoio Cartográfico, 1980 citado po: Calvente, 1980: 27), correspondendo a um " $r$ " de 1,27786, e a um tempo de duplicação de apenas 0,54 anos Fora de dúvida Rondônia é uma região onde c processo de desmatamento pode ser esperado a se revelar mais claramente, com áreas passando atualmente por rápidas mudanças em todos os estágios do processo: desde áreas ainde não ocupadas por Luso-Brasileiros (distinguidos dos Amerindios), até áreas onde a terra toda é revindicada por colonos e fazendeircs, e em que o desmatamento já é bastante avançado.

\section{Crescimento das áreas desimatadas}

Examinação de umá mais completa sérietempo de informações sobre desmatamento de uma área em Rondônia dará uma idéia melhor da natureza do padrão de crescimento das áreas desmatadas, ainda que os dados disponíveis sejam poucos. A informação veio de desenhos a partir de imagens LANDSAT tomadas em 1973, 1975, 1976 e 1978 da área mostrada na Figura 1. Os mapas estavam na escala de 1:250.000. com ligeiras variações (CV $=$ $0,59 \%$ ). As áreas desmatadas foram estimadas recortando as áreas desmatadas e pesando-as com uma precisão do $10^{-4} \mathrm{~g}$ (representando uma uma medida de $6,522 \times 10^{-2} \mathrm{~km}^{2}$ ). As

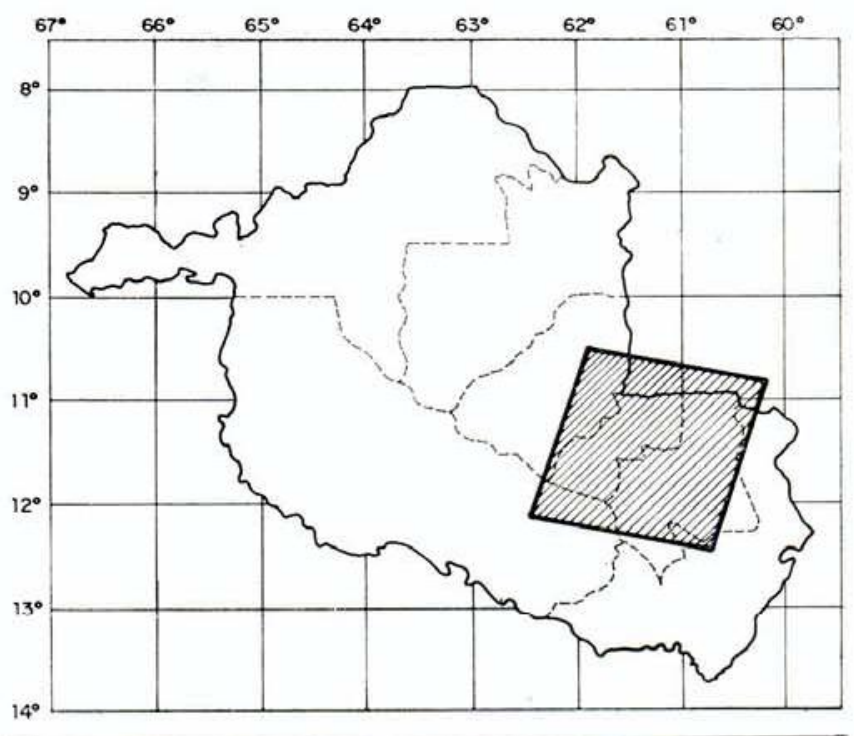

Fig. 1 - Mapa do Estado de Rondônia mostrando a área de cobertura das imagens LANDSAT (sombreado) e divisas dos municípios (linhas pontilhadas).

medidas foram tomadas separadamente em três projetos governamentais de colonização para pequenos produtores (Outo Preto, Jarú e Gy-Paraná), e duas áreas de fazendas de gado de até 3.000 ha, com algumas áreas de posseiros pequenos (Castro Alves e Gleba Corumbiara). O resultado do desmatamento é mostrado na Tabela 3.

Os dados revelam um rápido desmatamento em todas as cinco áreas. O desmatamento está avançado no projeto de colonização de Gy-Paraná, tendo atingido $15 \%$ da área até 1978. O desmatamento, expresso em porcentagem das áreas examinadas em cada projeto, é mostrado na Figura 2.

A. informaçăo acessível é inadequada para tirar conclusões inquestionáveis quanto às formas das curvas do desmatamento. No entanto, uma vez que esta é a mais longa série-tempo a que se tem acesso para este tipo de desmatamento, seria interessante examinar mais os dados disponíveis. A Tabela 4 compara as curvas linear e exponencial como representações dos dados para os três projetos para as quais desmatamento era constatada começando em 1973 assim como para o estudo de toda a área. $\left({ }^{2} \mathbf{j}\right.$

(2) - Deve frisar-se que desde que o desmatamento é um processo irreversível, as áreas desmatadas estão livres, apenas a variar com o tempo numa direção ascendente, assim violando a presunção de uma independente e normalmente distribuída variável dependente, requerida para as análises de regressão (Sokal \& Rohlf, 1969:409). 
TABELA 3 - Série-tempo do desmatamento em quatro áreas de assentamento de Rondônia

Ano

Desmatamento cumulativo $\left(\mathrm{km}^{2}\right)$

Desmatamento

Total

\begin{tabular}{lcccrrrr}
\hline & $\begin{array}{c}\text { Ouro } \\
\text { Preto }\end{array}$ & Jaru & $\begin{array}{l}\text { Castro } \\
\text { Alves }\end{array}$ & $\begin{array}{c}\text { Gy- } \\
\text { Paraná }\end{array}$ & $\begin{array}{c}\text { Gleba } \\
\text { Corumbiara }\end{array}$ & $\mathrm{Km}^{2}$ & $\%$ \\
\hline 1973 & 18 & $(0)$ & $(0)$ & 235 & 29 & 282 & 1,59 \\
1975 & 31 & 4 & 26 & 373 & 45 & 479 & 2,70 \\
1976 & 40 & 26 & 78 & 772 & 115 & 1.019 & 5,73 \\
1978 & 61 & 24 & 134 & 1.108 & 218 & 1.543 & 8,69 \\
\hline $\begin{array}{l}\text { Total das áreas } \\
\text { examinadas }\end{array}$ & 810 & 250 & 2.305 & 7.358 & 7.049 & 17.772 & 100,00
\end{tabular}

examinadas

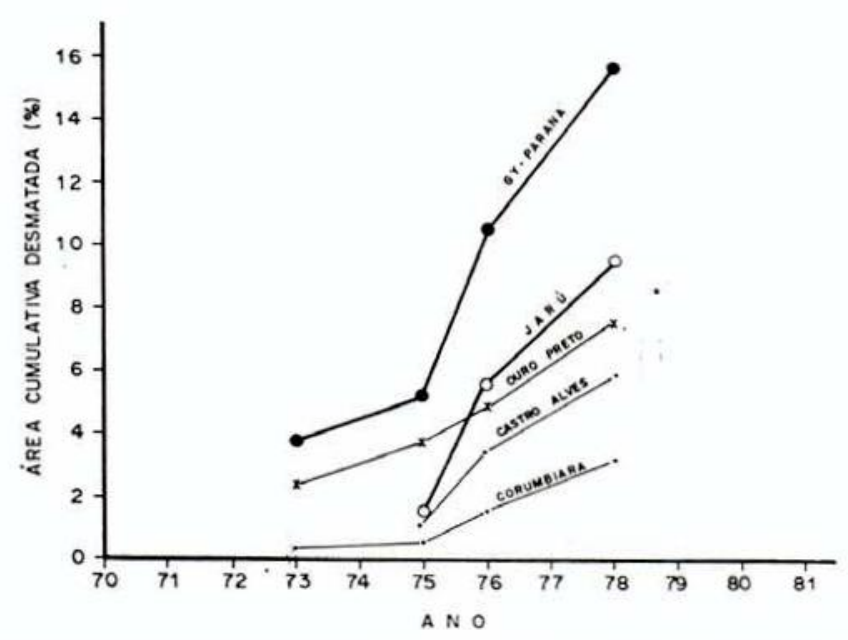

Fig. 2 - Desmatamento em 5 áreas de assentamento em Rondônia, a partir de medidas em imagens LANDSAT.

Ainda que desta pequena quantidade de dados a que se tem acesso não se possa tirar maicres conclusões, os coeficientes de determinação mais aitos indicam que a representação exponencial explica maior proporção da variança do que a linear, em todos os casos. A evidência que sugere a tendência exponencial não é de maneira alguma inequívoca, mas tal tendência levaria, se sustentada, a um completo desmatamento de todas estas áreas na presente década.

Qualquer tipo de processo de ajustamento simples de curvas, seja linear, exponencial ou outra, é destinado a provar-se inadequado por dar confiáveis projeções de um processo tão complexo quanto o desmatamento. $O$ que foi dito acima é uma advertência importante e eu a repetirei na conclusão deste artigo. Como primeiro passo para criar modelos mais realistas deste processo, o avanço do desmatamento está sendo examinado na atualidade em uma área de pequenos proprietários assentados sob os auspícios do governo em Rondônia e numa área sımilar da Rodovia Transamazônica, no Pará. Dentro de lotes individuais e dentro de blocos completamente ocupados destes lotes, o desmatamento não é exponencial. As médias cumulativas das áreas desmatadas aumentam aproximadamente linearmente, com algumas indicações de que o crescimento pode baixar depois de aproximadamente 6 anos de ocupação (Fearnside, s/d-c). Os dados discutidos acima, das imagens do LANDSAT sobre Rondônia, também mostram um desmatamento um pouco mais lento de 1976 a 1978 em comparação com o de 1975 a 1976. A corrente contínua de novos migrantes, assim como a parte substancial do desmatamento na região representado por fazendas e outras grandes empresas, assegura que a tendência ao nível regional continuará mostrando um crescimento acelerado das áreas desmatadas, sob as atuais condições. Eventualmente a taxa de desmatamento deve baixar, entretanto não há razão para acreditar que isso irá ocorrer de maneira simétrica e suave seguindo, por exemplo, uma curva de crescimento logístico.

\section{EXPECTATIVAS PARA O FUTURO}

Não se pode prever com clareza as taxas de desmatamento futuro. Muitas tendências 
TABELA 4 - Ajustamento linear e exponencial dos séries-tempo do desmatamento de Rondônia"

Item LINEAR

Coeficiente de determinaçăo

Coeficiente de regressão

Declives

Anos para completar o desmatamento

Ano do desmatamento completo

Ouro Preto

Gy-Paraná

Gleba Corumbiara

Total

da área em estudo

0,98

16,0

8,62

92,1

2066

0,93

0,89

4,2

39,04

183,23

39,3

180,5

2013

2154

0,93

172,6

263,27

66,9

2040

\section{EXPONENCIAL}

Coeficiente de determinação

Coeficiente de regressão

Taxa inata de crescimento ( $r$ )

Anos para completar o desmatamento

Ano do desmatamento completo

$\begin{array}{cc}1,00 & 0,94 \\ 18,5 & 231,5 \\ 0,24452 & 0,32619 \\ 15,5 & 10,6 \\ 1989 & 1984\end{array}$

0,94

0,95

26,2

287,9

$0,42401 \quad 0,35588$

13,2

11,7

1987

1985

(.) - Desmatamento em km2, do tempo em anos desde 1973. 
globais indicam um provável aumento do desmatamento da Amazônia no futuro. O fim iminente da floresta tropical úmida do Sudeste Asiático (Routley \& Routley, 1977) irá indubitavelmente levat a um aumento substancial na pressão dos interesses madeireiros internacionais, atualmente muito mais ativos no Sudeste Asiático do que na Amazônia. Depende muito da política do governo. O processo de desmatamento é muito difícil de controlar por razōes culturais, institucionais e práticas (Fearnside, 1979). A política do governo poderá afetar o desmatamento através da esco!ha e localização de agro-ecossistemas encorajados em desenvolvimentos influenciados por planejadores (Fearnside, s/d-b). As políticas que afetam o tamanho da população humana, e a distribuição de renda e posse da terra também têm, potencialmente, $\mathrm{u} \mathrm{m}$ a poderosa influência (Fearnside, s/d-a). As decisões do governo, afetando a economia em gerai, podem ter grande impacto sobre o desmatamento. As altas taxas de inflação no Brasil, $112 \%$ em 1980. proporcionaram forte motivação para os especuladores investirem em terias na Amazônia, elevando os preços a níveis acima daqueles que a terra poderia justificar, estritamente comı um insumo da produção agrícola. O desmatamento segue-se então para assegurar a revindicação do especulador sobre a terra (Fearnside, 1979). Tendências em outras regiões do Brasil afetando migração para a Amazônia incluem a produção, encorajada pelo governo, do álcool canavieira e ainda, mais importante, o financiamento da substituição de plantações de café, intensivos de mão-de-obra, por produção mecanizada da soja e trigo.

A taxa de desmatamento está estreitamente ligada à construção de estradas, atividade assumida grandemente pelo governo. A relação entre desmatamento e construção de estradas não é, provavelmente, uma simples reação de causa e efeito em qualquer direção, mas uma espécie de coevoluçâo entre as duas, como um processo de retroalimentação positiva. Na medida em que as estradas vão sendo construidas ou amelhoradas, e as estradas vincinais vão sendo acrescentadas, mais migrantes vão chegando na região, e os já presentes aumentam dramaticamente os índices de desmatamento; ao mesmo tempo a presença dos migrantes cria pressões, justificando as despesas para construir ainda mais e melhores estradas. Para 1982 há planos de asfaltar a Rodovia Cuiabá-Porto Velho (BR-364) e de aumentar a rede de estradas vincinais, com financiamento do Banco Mundial. O asfalto levará, sem dúvida, a um desmatamento mais rápido em Rondônia assim como nas áreas a que os migrantes estão se dirigindo a partir de Rondônia, como o Acre, Amazonas e especialmente Roraima.

Outra área em que a influência do governo é muito grande e a dos incentivos fiscais (Mahar, 1979; Fearnside, 1979). A Superintendência do Desenvolvimento da Amazônia (SUDAM) suspendeu recentemente novos incentivos para fazendas na parte da região oficialmente conhecida como floresta alta. Esta ação é encorajosa como uma indicação das intenções oficiais, mas o impacto sobre as taxas de desmatamento pode não ser tão grande como poderia ser pensado. Na escala da Amazônia Legal, está insenta de restrição a grande área classificada como "floresta de transição", onde estão localizadas a maioria das fazendas iilcentivadas pela SUDAM. Na área da floresta alta, os projetos pecuários aprovados pela SUDAM continuam a receber incentivos. Desde que a maioria destas fazendas desmataram apenas uma estreita fração da área total, muito desmatamento ainda terá lugar na floresta alta com a ajuda dos incentivos da SUDAM. Deve ser lembrado aqui também que antes da restrição a novos incentivos quase a metade do desmatamento nas zonas pecuárias foi feito sem os benefícios de nenhum incentivo: em 445.843 ha de desmatamento na área da Rodovia Belém-Brasília, levantados por Tardin et al. (1978:19), 45,4\% foram desmatados sem incentivos.

As taxas de desmatamento seriam afeta das pelos muitos aspectos das políticas de desenvolvimento contidas sob o projeto de lei da política florestal brasileira. A versão original desta lei foi concebida por uma comissão interministerial em 1979. Alegadamente, o conteúdo deste projeto de lei foi subseqüentemente alterado para remover restrições ao desmatamento da floresta alta para pecuária 
(Kerr, 1980). O representante na comissão interministerial do Conselho Nacional de Pes quisas Científicas e Tecnológicas (CNPq) notifica que o projeto de lei foi adiado, posteriormente, também em resposta aos interesses pecuários (A Crítica (Manaus), 01 de julho de 1981). Em junho de 1981 o Ministro do Interior do Brasil anunciou em Manaus que o projeto de lei da Política Florestal seria apresentado dentro em breve no Congresso Nacional.

\section{Conclusões}

1.) O desmatamento está ocorrendo rapida. mente. Existem algumas indicações, baseadas nas tendências em áreas de pequenas propriedades em Rondônia de 1973 a 1978, que as áreas desmatadas podem crescer exponencialmente, embora os dados disponíveis sejam in. suficientes para uma conclusão mais segura.

Aspectos do sistema consistente com um padrão exponencial incluem uma provável relação de retroalimentação positiva entre a construção de estradas e o desmatamento. Não se pode esperar que uma equação simples algébrica represente adequadamente 0 processo de desmatamento.

2.) As taxas de desmatamento variam grandemente em diferentes partes da região. Rondônia é o Estado em cuja extensão se processa à taxa mais alta.

3.) Nas propriedades individuais e nos grupos de propriedades já compietamente ocupadas, o desmatamento não é exponencial. A taxa de crescimento mais acelerada nas áreas de assentamento deve-se em grande parte à corrente de migrantes, que é provável aumentar.

4.) Assumindo uma tendência exponencial, a maioria das partes da Amazônia poderá ser desmatada até o final do século, e algumas regiōes, como Rondônia, por exemplo, ainda na presente década. Enfatizou-se que a tendência atual não necessita continuar: ela pode variar em grau ascendente ou descendente. Servem como ilustração, e não como previsão, os cálculos sobre as áreas desmatadas para o futuro.

5.) Muitas decisões do governo irão afetar as taxas de desmatamento na região.

6.) Mais importante do que saber a data pela qual a tendência atual pode levar a um completo desmatamento da região, seja mais cedo ou mais tərde, é a proteção das áreas designadas a não serem desmatadas, e o uso adequado das áreas designadas para desmatamento, para o que: 1.) são empregados agroecossistemas socialmente e economicamente sustentáveis, 2.) a concentração de renda e de posse da terra é limitada, 3.) o consumo total é limitado, e 4.) a população é mantida abaixo de sua capacidade de suporte, definido para incluir um adequado e suficientemente seguro padrão de vida. $\left({ }^{3}\right)$

\section{SUMMARY}

Deforestation in the Brazilian Amazon is proceeding at an unprecedented rate. Felling intensity is very uneven, being concentrated in certain parts of the region. A series of LANDSAT satellite images from settlement areas in Rondônia, from 1973 through 1978. indicate that clearing trends may be exponential, but information is too scant for firm conclusions. Available information from LANDSAT images from 1975 and 1978 covering all of Brazil's $5 \times 10^{6} \mathrm{~km}^{2}$ Legal Amazon indicate that if clearing were to proceed exponentially at the rate implied by the images at these two dates, the entire region would be cleared by 1991 . The unevenness of clearing rates would result in some areas being cleared sooner and others later; if clearing rates within each state or territory were to remain constant, they would be cleared at dates ranging from 1988 for Rondônia to $\mathbf{2 1 5 9}$ for Amapá. It is emphasized that none of these calculations represent forecasts of what is expected to happen, but only hypothetical scenarios of what would happen were these rates to continue. Deforestation is far too complex a process to be adequately represented by simple equations.

(3) - Agradeço à Secretaria de Planejamento do Territó rio Federal de Rondônia as cópias das imagens LANDSAT usadas para avaliar o desmatamento no território, e ao Instituto Nacional de Colonização e Reforma Agrária (INCRA) os mapas das áreas de colonizaçăo. A estimativa da área desmatada em Rondônia foi feita para o Projeto Estimativa de Capacidade de Suporte Agro-Ecossistemas Amazônicos/INPA pela Topografia Schuster Ltda., Manaus. O suporte financeiro veio do Projeto do Trópico Úmido (PTU) do Conselho Nacional de Pesquisas Científicas e Tecnológicas $(\mathrm{CNPq})$. Toda responsabilidade recai sobre o autor. Agradeço à direção da Interciencia pela permissão de publicar esta tradução (Fearnside, 1982). 
Forces leading to continued exponential growth of cleared areas include a probable positive feedback relationship between roadbuilding and deforestation. Many government policies have potential effects on clearing rates, including highway construction and improvement programs, agriculture and ranching incentives, and policies affecting population size, distribution of income, land tenure, and agricultural mechanization both in the Amazon and in source areas of migrants. Brazil's Forest Policy draft law, to cover many aspects of development policy in Amazonia, is a major unknown factor in future deforestation trends.

More important than the precise time remaining ror the bulk of Amazonia's rainforests are the designation and defense of areas to be left in forest, and the establishment in present and future cleared areas of agroecosystems which are agronomically and socially sustainable, limitation of concentration of income and land tenure, retriction of total consumption, and maintenance of human population levels below carrying capacity, defined to include an adequate and sufficiently cortain standard of living.

\section{REFERENCIAS BIBLIOGRAFICAS}

BRASIL. Conselho Nacional de Pesquisas Científicas e Tecnológicas. Instituto Nacional de Pesquisas Espaciais (CNPq-INPE). Departamento de Sensoriamento Remoto. Divisão de Aquisição de Dados. Seção de Aeronave e Apoio Cartográfico.

1980 - Dados sobre desmatamento. São José dos Campos/SP., INPE.

BRASIL. Território Federal de Rondônia. Secretaria de Agricultura.

1980 - Sistema Agrícola de Rondônia para o pequeno produtor. Porto Velho, Secretaria de Agricultura. 5 vols.

CALVENTE, A.T.

1980 - Formações não capitalistas no movimento de ocupação da Amazônia : Colonização agrícola em Rondônia - 1970/1980. Tese de mestrado em Economia. Brasília, Universidade de Brasília. 184p.

A CRITICA

1981 - "Ninguém provou venda da Região Amazô. nica”. Manaus, 01 de julho, cad. 1, p. 3.

FEARNSIDE, P.M.

1979 - The development of the Amazon Rain Forest: priority problems for the formulation of guidelines. Interciencia, 4 (6): 338 342 .

1982 - Deforestation in the Brazilian Amazon: How fast is it occurring? Interciencia, 7 (2): 82 88.

s/d. - Carrying Capacity for Human Populations: Colonization of the Brazilian Rainforest. (No prelo). s/d. - Development alternatives for the Brazilian Amazon: an Ecological Evaluation. (No prelo/Revista Interciencia).

s/d. - Land clearing behaviour in small farmer settlement schemes in the Brazilian Amazon and its relation to human carrying capacity. Trabalho apresentado no Simpósio "The Tropical Rainforest: Ecology and Resource Managment, Leeds. U.K.; 14-19 April (em preparação) .

JORNAL DO BRASIL

1977 - "Amazônia: o verde esconde um areal esteril”. Rio de Janeiro, 26 de junho, p. 7.

KERR, W.E.

1980 - Mudanças possíveis na política econômica que colaborariam para deixar a floresta em paz. Mensagem pronunciada ao Projeto Jaraqui em 10/05/80. Manaus, 8p. (A ser publicado manuscrito).

MAHAR, D.J.

1979 - Frontier development policy in Brazil: a study of Amazonia. New York, Praeger. 182p.

MUTHOO, M.K.

1977 - Perspectivas e Tendências do Setor Florestal Brasileiro, 1975 a 2000. Brasília, Instituto Brasileiro de Desenvolvimento Florestal. 2 vols. (Série Técnica, 8).

MYERS, N.

1980 - Conversion of Tropical Moist Forests. Washington, D.C., National Academy of Sciences. 205p.

PENTEADO, A.R.

1967 - Problemas de colonização e de uso da terra na região Bragantina do Estado do Pará. Belém. 488p.

PIELOU, E.C.

1974 - Population and community ecology: Principles and methods. New York, Gordon and Breach Science Publishers, 424p.

REIS, M.S.

1978 - Uma definição técnico-política para o aproveitamento racional dos recursos florestais da Amazônia brasileira. Conferência proferida durante o III Congresso Florestal Brasileiro, Manaus, 04-07 de dezembro. Projeto de Desenvolvimento e Pesquisa Florestal (PRODEPEF)/Instituto Brasileiro de Desenvoivimento Florestal (IBDF). Brasília, $21 p$.

ROUTLEY, R. \& ROUTLEY, V.

1977 - Destructive forestry in Australia and Melanesia. p. 374-97. In: Winslow, J.M. (compilador). The Melanesian Environment. Austrália, Australian National University Press. 562p. 
SERRÃO, E.A.S.; FALESI, I.C.; VIEGA, J.B. de \&

TEIXEIRA NETO, J.F.

1979 - Productivity of cultivated pastures on low fertility soils in The Amazon of Brazil. p. 195-225. In: Sánchez, P.A. \& Tergas, L.E. (compiladores). Pasture Production in Acid Soils of the Tropics: Proceedings of a Seminar held at CIAT Cali, Colombia 17-21 April, 1978: Series 03 EG-05. Colombia, Centro Internacional de Agricultura Tropical. $488 p$.

SIOLI, H.

1973 - Recent human activities in the Brazilian Amazon Region and their ecological effects. p. 321-34. In: Meggers, B.J.; Ayensu, E.S. \& Duckworth, W.D. (compiladores). Tropical Forest Ecosystems in Africa and South America: A comparative Review. Washington, D.C. Smithsonian Institution Press. 530p.

1980 - Foreseeable consequences of actual development schemes and alternative ideas. p. 257-68. In: Barblra-Scazzocchio, F. (compiladora). Land, People and Planning in Comtemporary Amazonla. Centre of Latin American Studies Occasional Publication n. 3. Cambridge, Cambridge University. 313p.

SOKAL, R.R. \& ROHLF, F.J.

1969 - Biometry; the principles and practice of statistics in Biological Research. San Francisco, E.U.A. W.H. Freeman. 776p.
TARDIN, A.T.; SANTOS, A.P. dos; LEE, D.C.L.; MAIA, F.C.S.; MENDONÇA, J.J.; ASSUNÇĀO, C.V.; RODRIGUES, J.E.; MOURA ABDON, M. de; NOVAES, R.A.; CHEN, S.C.; DUARTE, V. \& SHIMABUKURO, Y.E.

1979 - Levantamento de áreas de desmatamento na Amazônia Legal através de Imagens de Satělite LANDSAT. INPE-COM3/NTE. S. José dos Campos/SP. 3p.

TARDIN, A.T.; SANTOS, A.P. dos; MORAIS NOVO, E.M.I. \& TOLEDO, F.L.

1978 - Projetos agropecuários da Amazônia; desmatamento e fiscalização - relatório. A

Amazônia Brasileira em Foco, n. ${ }^{\circ}$ 12, p. 7-45.

TARDIN, A.T.; LEE, D.C.L.; SANTOS, R.J.R.; ASSIS, O.R. de; SANTOS BARBOSA, O.R. dos; LOURDES MOREIRA, M. de; PEREIRA, M.T.; SILVA, D. \& SANTOS FILHO, C.P. dos.

1980 - Subprojeto Desmatamento, Convênio IBDF/ CNPq-INPE 1979. Instituto Nacional de Pes-

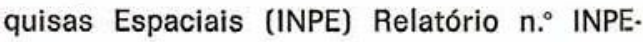
1649-RPE/103. São José dos Campos/SP., INPE. $44 \mathrm{p}$.

(Aceito para publicação em $30 / 04 / 82$ ) 